\title{
Mars 2020 Entry, Descent and Landing Instrumentation (MEDLI2)
}

\author{
Deepak Bose, ${ }^{1}$ Henry Wright, ${ }^{2}$ Todd White, ${ }^{3}$ Mark Schoenenberger, ${ }^{2}$ Jose Santos ${ }^{4}$ Chris \\ Karlgaard, ${ }^{2}$ Chris Kuhl, ${ }^{2}$ Tomo Oishi, ${ }^{4}$ and Dominic Trombetta ${ }^{2}$ \\ ${ }^{1}$ NASA Ames Research Center, Moffett Field, CA 94035, USA \\ ${ }^{2}$ NASA Langley Research Center, Hampton, VA 23681, USA \\ ${ }^{3}$ AMA, Inc, Moffett Field, CA 94035, USA \\ ${ }^{4}$ Jacobs Technology, Moffett Field, CA 94035, USA
}

\section{Introduction}

This paper will introduce Mars Entry Descent and Landing Instrumentation (MEDLI2) on NASA's Mars2020 mission. Mars2020 is a flagship NASA mission with science and technology objectives to help answer questions about possibility of life on Mars as well as to demonstrate technologies for future human expedition. Mars2020 is scheduled for launch in 2020. MEDLI2 is a suite of instruments embedded in the heatshield and backshell thermal protection systems of Mars2020 entry vehicle. The objectives of MEDLI2 are to gather critical aerodynamics, aerothermodynamics and TPS performance data during EDL phase of the mission. MEDLI2 builds up the success of MEDLI flight instrumentation on Mars Science Laboratory mission in 2012. MEDLI instrumentation suite measured surface pressure and TPS temperature on the heatshield during MSL entry into Mars. MEDLI data has since been used for unprecedented reconstruction of aerodynamic drag, vehicle attitude, in-situ atmospheric density, aerothermal heating, transition to turbulence, in-depth TPS performance and TPS ablation. [1,2] In addition to validating predictive models, MEDLI data has highlighted extra margin available in the MSL forebody TPS, which can potentially be used to reduce vehicle parasitic mass.

MEDLI2 expands the scope of instrumentation by focusing on quantities of interest not addressed in MEDLI suite. The type the sensors are expanded and their layout on the TPS modified to meet these new objectives. The paper will provide key motivation and governing requirements that drive the choice and the implementation of the new sensor suite. The implementation considerations of sensor selection, qualification, and demonstration of minimal risk to the host mission will be described. The additional challenges associated with mechanical accommodation, electrical impact, data storage and retrieval for MEDLI2 system, which extends sensors to backshell will also be described.

\section{Goals and Objectives}

NASA's exploration and technology roadmaps call for capability advancements in Mars entry, descent, and landing (EDL) systems to enable increased landed mass, a higher landing precision, and a wider planetary access. It is also recognized that these ambitious EDL performance goals must be met while maintaining a low mission risk in order to pave the way for future human missions. As NASA is engaged in developing components of future EDL systems and technologies via testing at Earth, flight instrumentation such as MEDLI and MEDLI2 on existing Mars missions is providing valuable engineering data for performance improvement, risk reduction, and an improved definition of entry loads and environment.

The following goals identified for MEDLI2.

1) Acquire flight data to define entry aerothermal environment

2) Acquire flight data to reduce entry vehicle TPS mass

3) Acquire flight data to improve Aerocapture and EDL performance

The abovementioned goals for MEDLI2 will be met via reconstruction of aerothermal environment, aerodynamic performance, and TPS in-depth performance from the sensor data. Specific objectives for each 
discipline have been developed for maximal impact on the goals. These objectives will drive the top level instrument requirements to be described later in the paper. For aerothermal and TPS goals the objectives are defined such that they reduce design margins and prediction uncertainty. For aerodynamics goals, the objectives are defined similarly to reduce uncertainty and enable validation of the aerodynamic database.

\section{Science Requirements}

A set of top-level science requirements are defined to set reconstruction and measurement targets for MEDLI2. The requirements are then distilled further to ensure that sensor accuracy, measurement range, and data sampling rate are adequate to meet MEDLI2 objectives. A concise list science requirements are shown below.

Aerothermodynamics and TPS:

1) Reconstruct forebody aeroheating

2) Determine forebody TPS temperatures

3) Reconstruct afterbody aeroheating

4) Measure afterbody heat flux

Aerodynamics:

5) Reconstruct supersonic aerodynamic axial force

6) Reconstruct wind relative vehicle attitude

7) Reconstruct atmospheric density and winds

The lower level instrument requirements also drive sensor selection and layout, among several implementation details.
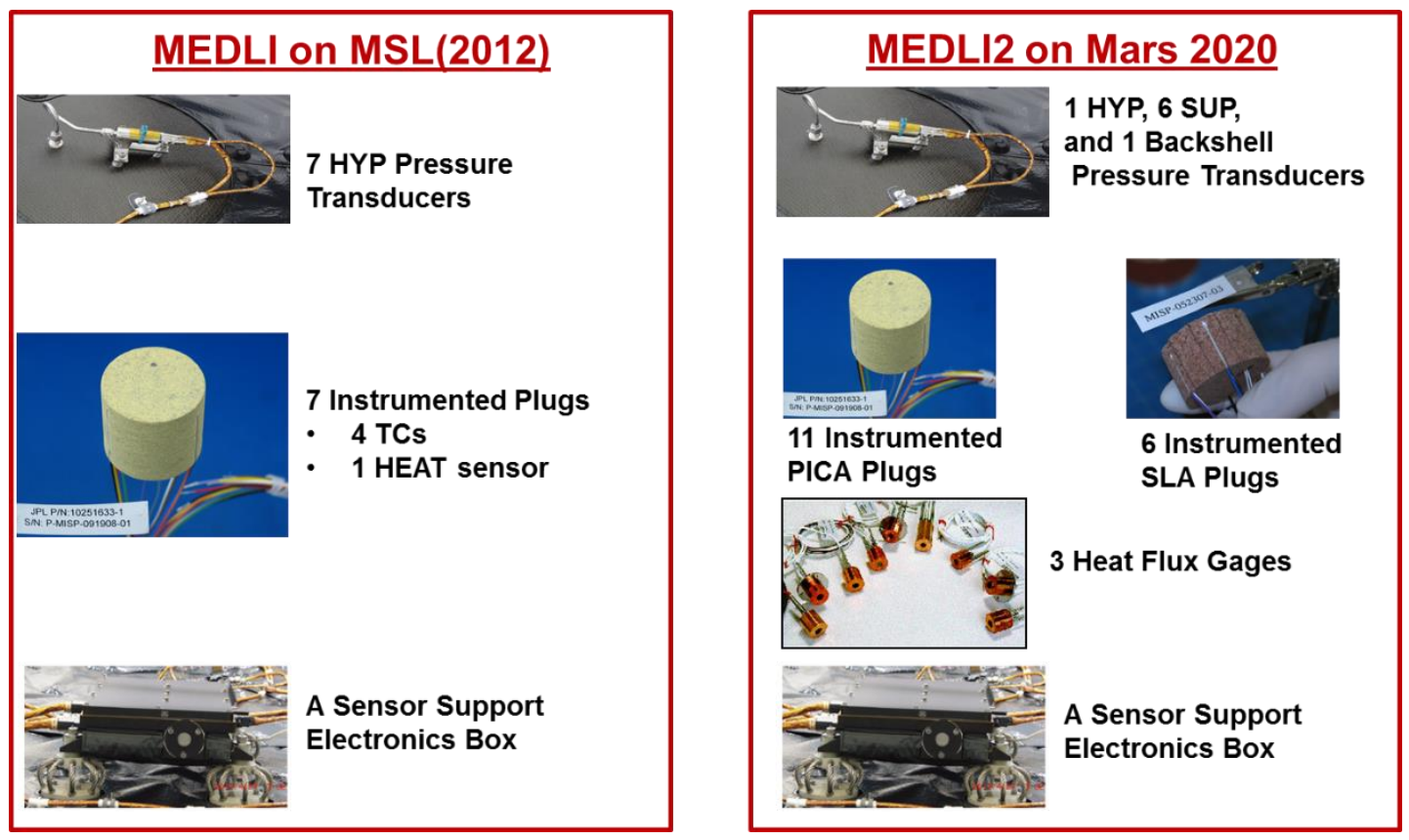

Figure 1. MEDLI and MEDLI2 instrument suite

\section{Sensor Selection and Layout}

Figure 1 shows a sample of instruments under consideration for MEDLI2 suite. MEDLI instrumentation suite is also shown for comparison. Similar to MEDLI, pressure transducers and thermocouples form a bulk of the 


\section{Abstract submitted to the 46th AIAA Thermophysics Conference}

instrumentation. MEDLI2 however has additional sensor types such has heat flux sensors, instrumented SLA$561 \mathrm{~V}$ plugs for backshell, and lower pressure transducers for supersonic flight and backshell measurements.

Pressure Transducers:

In order to meet the science objectives, three types of pressure transducers are being selected. Each type of transducer will be calibrated for a specific measurement range and required accuracy. Hypersonic transducers, identical to the ones used in MEDLI, will measure surface pressure from 0-5 psi that will span entire test period including the peak dynamic pressure environment. However, these transducers do not provide sufficient accuracy at lower pressures expected during supersonic flight. A separate set of supersonic transducers are, therefore, being considered that are accurate over the 0-1 psi range. A third type of pressure transducer is also being selected to measure ultra-low pressure of 0-0.1 psi expected on the backshell of the vehicle. The layout of the sensors on the heatshield and backshell are shown in Fig. 2. The locations of the pressure transducers on the forebody are defined using an optimization algorithm that minimizes errors in reconstruction of vehicle angle of attack and side-slip.

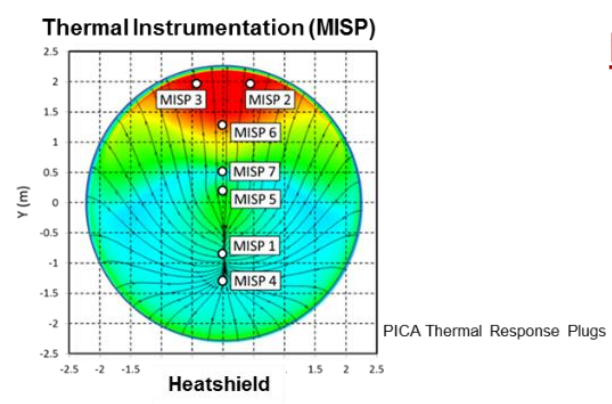

MEDLI (2012)
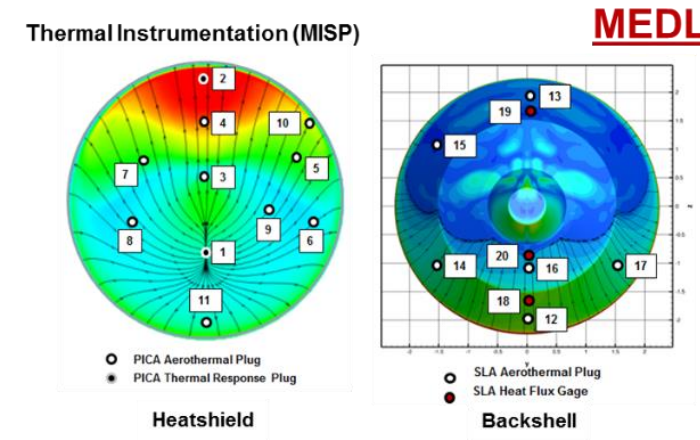

MEDLI2 (2020)
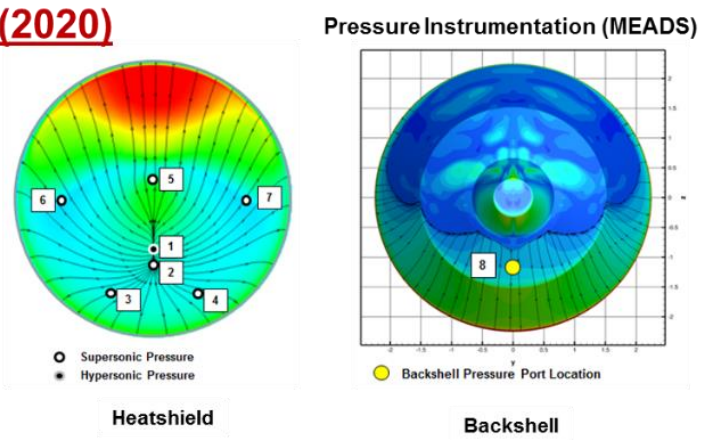

Figure 2. MEDLI and MEDLI2 sensor locations on Mars 2020 heatshield and backshell.

Thermocouples:

Thermocouples (TCs) will embedded in the TPS using instrumented plugs as shown in Fig. 1. Unlike MEDLI, MEDLI2 TCs will focus on near surface temperatures. Two types of TCs will be used. Type-R (or S) will be used at shallower depths $(<0.1$-in) and Type-K will be used at greater $(\geq 0.1$-in $)$ depths. MEDLI2 TC layout will also emphasize reconstruction of aeroheating over a larger area of the TPS in order to capture turbulent footprint and transition phenomena, which results in an increased number of TC plugs as shown in Fig. 2. Three types of plugs are chosen. The Aerothermal plugs consisting of only one TC will map the aerothermal environment on the forebody and backshell. The Thermal Response plugs will contain three TCs and measure in-depth response of the TPS material. All forebody plugs will be made from PICA, which is the forebody TPS. The afterbody plugs will be made from SLA-561V, the backshell TPS, and will contain one TC each. The afterbody plugs will map the aerothermal environment in the attached and separated flow regions of the backshell, and also span both conical surfaces. The parachute cone cover will not be instrumented due to implementation challenges. 
Heat Flux Sensors and Radiometer:

The lower levels of heating $(<25 \mathrm{~W} / \mathrm{cm} 2)$ predicted in the backshell opens a larger set of options for more accurate sensors for direct measurement of heat flux, and separate measurement of radiative heating. MEDLI2 is currently testing candidate heat flux sensors and radiometers for accuracy and survivability. These sensors form a new suite of instrumentation for Mars entry vehicle. In order to bring an appropriate sensor technology to flight readiness, a qualification program is being undertaken. The qualification will include thermal and mechanical survivability under flight-relevant aerothermal, shock, vibration, and thermal vacuum conditions. The candidate locations for these sensors are shown in Fig. 2. The locations are defined to measure convective and radiative environments on windside attached flow as well as on the leeside separated flow regions.

\section{System Architecture and Operation}

MEDLI2 sensors will be controlled by one sensor support electronics (SSE) box. The SSE box mounted on the inside of the heatshield will perform all supporting functions: sensor signal conditioning, excitation, multiplexing, analog-to-digital conversion, cold-junction compensation, power management, and data communication. Cable harness for power, data communication, and sensor output will form a network throughout the vehicle, passing through a cable cutter at the heatshield separation interface. The data sampling rate will vary depending on the sensor type. A typical sampling rate is expected to be $8 \mathrm{~Hz}$. The complete MEDLI2 dataset will be stored in the Rover Compute Element, and will be transferred back to earth upon landing.

MEDLI2 will be turned on, and will begin collecting data 10 mins prior to entry interface. MEDLI2 system will continue collecting data during the critical test period of hypersonic and supersonic portions of the trajectory. MEDLI2 system will turned off shortly before the heatshield is jettisoned, which will occur after parachute deployment and eventual deceleration to subsonic speeds.

\section{Reconstruction Targets}

MEDLI2 science objectives will rely on successful reconstruction of aerodynamic, aerothermal, and TPS performance using sensor data and physics based modeling. The physics based models are often necessary to bridge the gap between sensor measurements and quantities of interest. Table I presents the quantities of interest and targeted reconstruction accuracy. In the final paper an error budget will be presented that demonstrates the allocation of errors to sensor measurements and physics based models.

\begin{tabular}{lll}
\hline Quantity of Interest & Reconstruction Target & Relevant Sensors \\
\hline Forebody Reconstructed Heating & $\pm 15 \mathrm{~W} / \mathrm{cm} 2$ & Forebody TCs \\
\hline Boundary Layer Transition & \pm 1 second & Forebody TCs \\
\hline In-depth Temperatures & $\pm 50 \mathrm{C}$ & Forebody TCs \\
\hline Afterbody Reconstructed Heating & $\pm 3 \mathrm{~W} / \mathrm{cm}^{2}$ & Afterbody TCs \\
\hline Afterbody Heat Flux & $\pm 1 \mathrm{~W} / \mathrm{cm}^{2}$ & Heat Flux Sensor/Radiometer \\
\hline Axial Force Coefficient & $\pm 2 \%$ & Supersonic Press. Transducer \\
\hline Vehicle Attitude & \pm 0.5 degrees & Supersonic Press. Transducer \\
\hline Atmospheric Winds & $\pm 10 \mathrm{~m} / \mathrm{s}$ & Supersonic Press. Transducer \\
\hline Atmospheric Density & $\pm 5 \%$ & Hypersonic Press. Transducer \\
\hline Backshell Pressure & $\pm 4 \mathrm{~Pa}$ & Backshell Press. Transducer \\
\hline
\end{tabular}

\section{Conclusions}

This paper will introduce MEDLI2 instrumentation suite on Mars2020 heatshield and backshell designed to acquire critical EDL data. MEDLI2 will support key goals and objectives to improve aerodynamics, 
aerothermodynamics, and TPS response predictions and design margins. The science objectives will be distilled into instrument requirements that will determine sensor type and layout. The development and qualification challenges of the MEDLI2 will also be described. The paper will also present reconstruction techniques and accuracy targets via analysis of errors in sensor measurements and physics based models.

\section{References}

